\title{
Directly Identifying Phase Segregation in 2D Quaternary Alloys
}

Jordan A. Hachtel ${ }^{1}$, Sandhya Susarla ${ }^{2}$, Vidya Kochat ${ }^{2}$, Chandrasekhar Tiwary ${ }^{2}$, Pulickel Ajayan ${ }^{2}$, and Juan Carlos Idrobo ${ }^{1}$

${ }^{1 .}$ Center for Nanophase Materials Sciences, Oak Ridge National Laboratory, Oak Ridge, TN, USA

2. Department of Mechanical Engineering \& Materials Science, Rice University, Houston, TX, USA

Transition metal dichalcogenides (TMDCs) are one of the most studied family of 2D materials over the past few years, mostly due to significant advances in fabricating 2D TMDC heterostructures with highly variable optical, electrical, and physical properties [1,2]. In these heterostructures, controlling and/or tuning the important aspects of the material, such as the band gap or the conductivity, remains a constant challenge. Monolayer TDMC alloys can provide a high degree of control toward creating 2D materials with tailored responses.

Monolayer quaternary TDMC alloys having the $\mathrm{MX}_{2}$ stoichiometry, where $\mathrm{M}=(\mathrm{Mo}$ or $\mathrm{W})$ and $\mathrm{X}=(\mathrm{S}$ or Se), are predicted to be stable and have strong tunability of their optical properties [3] since the individual $\mathrm{MX}_{2}$ compositions possess a wide range of band gaps (e.g., $\mathrm{MoSe}_{2}-1.55 \mathrm{eV}, \mathrm{WSe}_{2}-1.65 \mathrm{eV}$, $\mathrm{MoS}_{2}-1.85 \mathrm{eV}$, and $\mathrm{WS}_{2}-1.95 \mathrm{eV}$ ). These monolayer $\mathrm{MX}_{2}$ alloys are produced using chemical vapor deposition, which provides tight control over the fabrication conditions in terms of temperature and precursor ratios, allowing us to produce quaternary alloys with a wide range of compositions.

Aberration-corrected scanning transmission electron microscopy (STEM) is used to determine the composition and phase distribution of quaternary 2D TMDC alloy layers. The Z-contrast imaging capabilities of high-angle annular dark field (HAADF)-STEM allows information about the chemical composition of the alloy to be extracted directly from the contrast in the STEM images.

Figure 1a shows a HAADF-STEM image of quaternary alloy, synthesized to have approximately equal amounts of Mo and $\mathrm{W}$ as well as $\mathrm{S}$ and Se. The individual atoms associated with the $\mathrm{X}$ - and M-sites are extracted based on the coordination of the atoms, and the image contrast/intensities are used to assign each atom in the image (i.e., for the X-sites three distinct intensities are observed, which are labeled as $\mathrm{S}_{2}, \mathrm{Se}_{2}$, and $\mathrm{SSe}$ ). The corresponding atom-labeled image, with the atom locations shown by different colors along with the total number of atoms in each atomic column, is shown in Figure $1 \mathrm{~b}$.

Evidence for phase segregation is seen in the HAADF-STEM image (Figure 1a), where regions of bright contrast are observed at the bottom-left and top-center of the image. The atom-labeled image (Figure 1b) provides additional insight by showing the composition of those regions. In Figure $1 \mathrm{~b}, \mathrm{~W}$ atoms are marked by red circles and $\mathrm{Se}_{2}$ columns are marked by orange circles; in the atom-labeled image (Figure 1b), nearly all the $\mathrm{W}$ atoms in the image reside in these high-contrast regions, and the X-site atoms consist of mainly of $\mathrm{Se}_{2}$. Furthermore, in the remainder of the atom-labeled image, the Mo bonds are shared equally with $\mathrm{S}_{2}, \mathrm{SSe}$, and $\mathrm{Se}_{2}$ indicating that Mo does not exhibit preferential bonding to a specific type of atom.

The analysis technique outlined above shows that the high-intensity regions correspond to local segregation of $\mathrm{WSe}_{2}$ from the rest of the 2D TMDC alloy, as the Mo does not have a strong bonding preference, while $\mathrm{W}$ demonstrates a strong preference to distribute within $\mathrm{Se}_{2}$ columns. The same 
analysis can be performed on larger field-of-views and the statistical relationship between the M-site and $\mathrm{X}$-site atoms can be quantified. The result is the ability to use aberration corrected STEM to directly observe phase segregation in alloyed heterostructures at the atomic level, and to correlate the effects of different growth conditions with the genuine structure of the alloys to improve the tunability of 2D TMDC alloys.

\section{References:}

[1] Q H Wang et al, Nature Nanotechnology 7 (2012), p. 699.

[2] Y Gong et al, Nature Materials 13 (2014), p. 1135.

[3] G Wang et al. Nature Communications 6 (2015) 10110

[4] Research conducted at ORNL's Center for Nanophase Materials Sciences, which is a U.S.

Department of Energy, Office of Science User Facility.
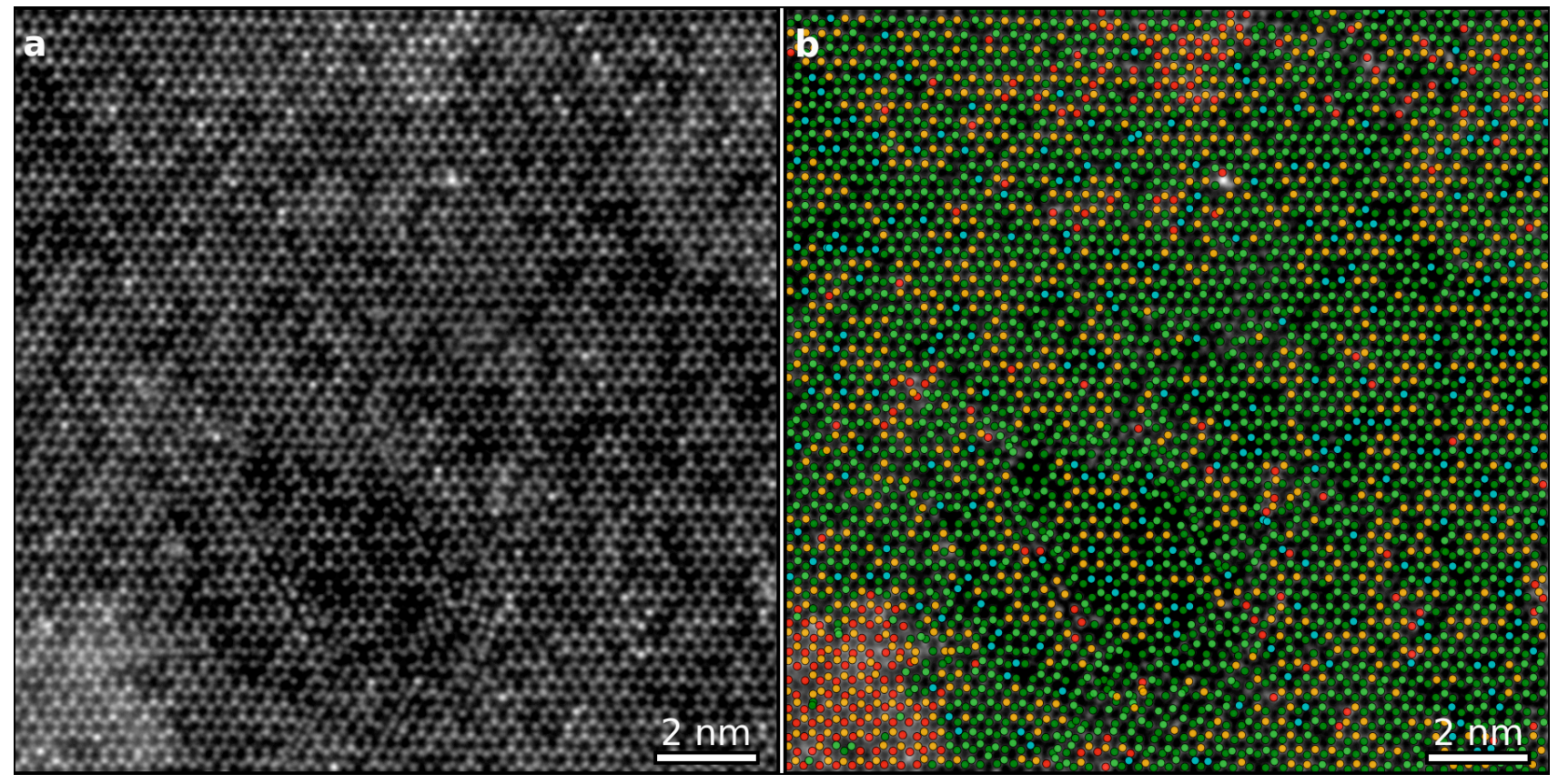

Figure 1. (a) HAADF-STEM image of a quaternary Mo-W-S-Se alloy. (b) Associated atom-labeled image (Green - Mo, Red - W, $\mathrm{S}_{2}-$ Cyan, SSe - Light Green, and $\mathrm{Se}_{2}$ - Orange) that demonstrates phase segregation of $\mathrm{WSe}_{2}$ from the remainder of the alloy structure. 